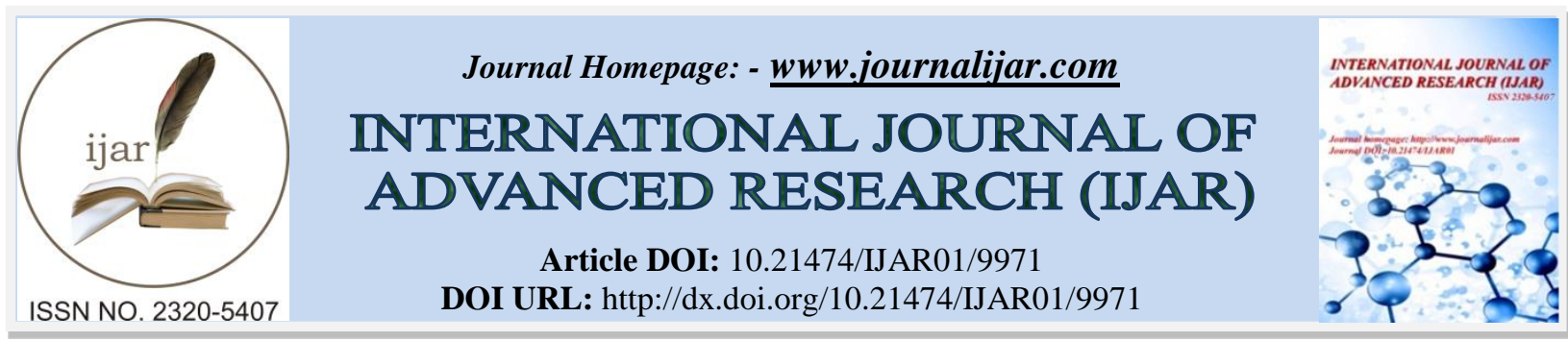

RESEARCH ARTICLE

\title{
LIPOPHILIC AND STRUCTURE ACTIVITY RELATIONSHIPS STUDY OF THIOSEMICARBAZONES AND DERIVATIVES.
}

Glinma Bienvenu ${ }^{1}$, Medegan Sèdami ${ }^{2}$, Yayi Eléonore ${ }^{1}$, Agnimonhan F. Hyacinthe ${ }^{1}$, Kpoviessi D.S. Salomé ${ }^{1}$, Quetin-leclercq Joëlle ${ }^{3}$, Accrombessi C. Georges ${ }^{1}$, Kotchoni O. Simeon ${ }^{4}$, Poupaert H. Jacques ${ }^{3}$ and Gbaguidi A. Fernand ${ }^{1,2}$.

1. Laboratoire de Chimie Organique Physique et de Synthèse (LaCOPS), Département de Chimie, Faculté des Sciences et Techniques (FAST), Université d'Abomey-Calavi, 01 BP 4521 Cotonou, Bénin.

2. Laboratoire de Chimie Organique Pharmaceutique, Ecole de Pharmacie, Faculté des Sciences de la Santé, Université d'Abomey-Calavi, Campus du Champ de Foire, 01 BP 188, Cotonou, Bénin.

3. Louvain Drug Research Institute (LDRI), School of Pharmacy, Université Catholique de Louvain, B1 7203 Avenue Emmanuel Mounier 72, B-1200 Brussels, Belgique.

4. Department of Biology and Center for Computational and Integrative Biology, Rutgers University, Camden, NJ 08102, USA.

\section{Manuscript Info}

Manuscript History

Received: 05 September 2019

Final Accepted: 07 October 2019

Published: November 2019

Key words:-

Synthesize,

trypanocidal, lipophilicity, selectivity.

\section{Abstract}

Traditionally, small molecules have been a reliable source for discovering novel biologically active compounds because these molecules are easily synthesized and their smooth structural optimization would usually lead to a feasible candidate compound. Here, some thiosemicarbazones, N(4)-methyl and N(4)-phenyl-3thiosemicarbazones were synthesized in good yield (52-84\%), characterized and then their anti-parasitic activity were evaluated. The structure and lipophilic-activity relationships of compounds were particularly studied. Among them, some products exhibited trypanocidal activity with their half inhibitory concentration $\left(\mathrm{IC}_{50} \leq 10\right.$ micromolar " $\mu \mathrm{M}$ ") especially compounds $\mathbf{L}_{\mathbf{1 - 3}}, \mathbf{D}_{\mathbf{2}}, \mathbf{B}_{\mathbf{3}}, \mathbf{C}_{\mathbf{3}}, \mathbf{D}_{\mathbf{1}}$ (from 2 to $8.73 \mu \mathrm{M}$ ). Other showed moderate antitrypanosomal activity with their $\mathrm{IC}_{50}$ between 12 to $87 \mu \mathrm{M}\left(\mathbf{L}_{\mathbf{4}}, \mathbf{C}_{\mathbf{2}}, \mathbf{C}_{\mathbf{1}}, \mathbf{B}_{\mathbf{2}}\right)$ while certain showed little activity $\left(\mathrm{IC}_{50} \geq 100 \mu \mathrm{M}\right)$. Some active products turned out quick selective on the parasite with their selectivity index greater than to unit (SI $\geq 1)$.

Several factors including lipophilicity, steric and electronic effects of the substituents have played a vital role in this activity. The elongation of the carbon chain of the carbonyl, the substitution on a phenyl radical, the fixing of a methyl or phenyl on the N(4) nitrogen atom induced significantly the increased trypanocidal activity of compounds. This is the case specifically of N(4)-methyl and especially of N(4)-phenylsubstituted thiosemicarbazones. Such compounds could be able to have applications in the treatment of parasitic diseases.

Copy Right, IJAR, 2019,. All rights reserved. 


\section{Introduction:-}

Nowadays, microbial and parasitic diseases are resistant to existing treatments. For these reasons, chemists have been made a great effort to the development of compounds with biological activity that will be used in pharmaceutical chemistry. Currently, most antiparasitic drugs are considered orphan drugs, with the main exception of antimalarials. The pharmaceutical considerations outweigh all others, because the economic return on the development of anti-parasitic drugs is limited. Therefore, it is necessary to find less expensive alternatives for the treatment of parasitic disease (Soates et al., 2011). Recently, due to having wide-spectrum biological activity of thiosemicarbazones derivatives that synthesis studies made, interest on these compounds has been considerably increased in the pharmaceutical sector at the present time (Rogolino et al., 2015 ; Büscher et al., 2017 ; Zani et al., 2017). Thiosemicarbazones and semicarbazones due to be a small molecule widely used in the treatment of antiviral, anticancer and antiparasital disease. More recently, it has been found that they are highly effective antiparasital compounds against Trypanasoma cruzi parasites that cause especially Malaria and Chagas diseases. Generally, thiosemicarbazones show this effect by causing the inhibition of cysteine proteases in this type of parasites and derivatives (Du et al., 2002 ; Beraldo and Gambino, 2004 ; Greenbaum et al., 2004 ; Fujii et al., 2005 ; Jeremy et al., 2008).

In the light of this important data which have been achieved with the literature survey considering that the thiosemicarbazones are biologically active compounds, synthesis of the thiosemicarbazone derivatives expected to show positive activity was carried out.

African trypanosomiases are still a serious health and economic problem that requires not only the application of the knowledge and resources currently available, but also their improvement through multidisciplinary research (Simarro et al., 2012 ; Grant et al., 2015). Animal trypanosomiasis is a major constraint for the livestock industry in developing countries (Amer et al., 2011). In East Africa, animal trypanosomiasis is caused by numerous protozoan parasites transmitted by tsetse flies, including Trypanosoma vivax, T. congolense and subspecies of $T$. brucei sl ( $T$. bruce $i$ and T. $b$ zoonotic rhodesiense infectious for humans) that can co-circulate in domestic and wild animals (Cox et al., 2010).

In this paper, we described the synthesize of thiosemicarbazones, N(4)-methyl and N(4)-phenyl-3thiosemicarbazones of benzaldehyde, 2'-methylacetophenone, propiophenone, benzophenone with its substituted derivatives. The compounds were tested for their antitrypanosomal activity against Trypanosoma brucei brucei and their toxicity on larvae shrimp Artemia salina Leach. And then, some structure-activity and lipophilicity-activity relationships were examined.

\section{Material And Methods:- \\ Reagents}

All reagents were obtained from chemical societies: Sigma-Aldrich, Acros Organic, Janssen Chimica, Prolabo and Riedel-de Haen. Substrates, reagents, catalysts and solvents were used directly for syntheses without any further purification. There are : benzaldehyde, 4'-methylacetophenone, benzophenone and derivate, hydrochloric acid $(\mathrm{HCl})$, glacial acetic acid (AAG), Technical ethanol (EtOH), thiosemicarbazone, 4-methyl-3-thiosemicarbzide, 4phenyl-3-thiosemicarbazide.

\section{Equipment}

All synthesized compounds were characterized by Nuclear Magnetic Resonance spectra using Bruker Avance 400. UltraSheild with dimethylsufoxide (DMSO) $-\mathrm{d}_{6}$ or chloroform $\mathrm{CDCl}_{3}$ and then Mass Spectrophotometer spectra obtained using the method of Atmospheric-pressure chemical ionization and mass is given in $\mathrm{m} / \mathrm{z}$ of $\left[\mathrm{MH}^{+}\right]$. The

frequencies for ${ }^{1} \mathrm{H}$ and ${ }^{13} \mathrm{C}$ are 400.130 and $100.612 \mathrm{MHz}$ respectively. Chemical shifts are given in parts per million $(\mathrm{ppm})$ relative to tetramethylsilane as internal standard. Multiplicity was designated as singlet (s), doublet (d), doublet dedouble (dd), triplet (t), quintuplet (qi) and multiplet (m). Melting points (m.p.) were determined on a fusionometer of the type electrothermal 1A 9000 and were not corrected.

\section{Methods}

\section{Synthesis of the compounds}

An equimolar mixture $(0.01 \mathrm{~mol})$ of thiosemicarbazide and analogues dissolved in $10 \mathrm{~mL}$ ethanol $\left(\mathrm{EtOH}^{\circ} 6^{\circ}\right)$ was added slowly to a solution $(0.01 \mathrm{~mol})$ of arylketone dissolved in $20-30 \mathrm{~mL}$ of EtOH in presence of acid $(\mathrm{HCl}, 1 \mathrm{~N}$ or 
GAA). The mixture was heated at reflux for $4 \mathrm{~h}$ with stirring. After cooling, the precipitate was filtered, washed with cold distilled water until neutrality, dried and then recrystallized in ethanol.

All compounds after synthesis have been submitted to the in vitro anti-trypanosomal test on the bloodstream form of the strain 427 of Trypanosoma brucei brucei and were evaluated for their in vitro cytotoxicity on Artemia salina Leach following standard biological methods.

\section{Pharmacology}

\section{Anti-trypanosomal test}

The assessment is performed on the bloodstream form of the strain 427 of Trypanosoma brucei brucei by the «LILIT Alamar Blue ${ }^{\mathrm{TM}}$ » method (Baltz et al., 1985 ; Räz et al., 1997). The stock solutions of each thiosemicarbozone have been prepared from an initial concentration of $10 \mathrm{mg} / \mathrm{mL}$ in dimethylsufoxide (DMSO). The trypanosomes are grown in a medium containing $10 \%$ of heat inactivated fetal calf serum and bloodstream form supporting factor. The trypanosome suspensions were adjusted to $5 \times 10^{4}$ tryp $/ \mathrm{mL}$. In each well, $50 \mu \mathrm{L}$ of different dilutions of the stock solution were added to $50 \mu \mathrm{L}$ of suspension of trypanosomes. The plates were then incubated at $37^{\circ} \mathrm{C}$ for 72 hours in an atmosphere with $5 \% \mathrm{CO}_{2} .10 \mu \mathrm{L}$ of dye "Alamar BlueTM" is added to each well and then incubated for 4 hours. The dye "Alamar BlueTM" is a reagent for detecting enzymatic activity. The wells in which the concentration of compound is insufficient to inhibit the proliferation of trypanosomes are stained. The halfinhibitory concentration is the concentration of unstained wells in which there is the lowest amount of thiosemicarbazones. The plate reading is made in comparison with control wells on a fluorescence plate reader using an excitation wavelength of $530 \mathrm{~nm}$ and an emission wavelength $590 \mathrm{~nm}$. We carried out the test in triplicate for each compound. All data were expressed as means \pm standard deviation of triplicate measurements.

\section{Cytotoxicity screen}

The cytotoxicity test was performed on larvae of brine shrimp (Artemia salina Leach) by the method of Sleet and Brendel (1983). Artemia salina eggs were incubated in seawater until hatching of young larvae (48 hours). Then, series of solutions of test compound at varying concentrations were prepared in DMSO/seawater. A defined number of larvae were introduced into each solution and incubated under rocking condition for $24 \mathrm{~h}$. To evaluate the toxicity of the solution, counting of larvae viability was performed under microscope by determining the number of dead larvae in each solution. In the case where there was death in the control medium, the data was corrected by Abbott's formula:

$\%$ death $=[($ nd test - nd control $) /$ nd control $)] \times 100($ Abott, 1925) with nd $=$ number of dead larvae.

Data (dose-response) were transformed by logarithm and the halflethal concentration $\mathrm{LC}_{50}$ was determined by linear regression (Hafner et al., 1977). Tests were carried out in triplicates. All data were expressed as mean \pm standard deviation of triplicate measurements.

\section{Results And Discussion:-}

\section{Results}

\section{Chemistry}

Before synthesizing the compounds, we carried out a theoretical study based on the pharmacokinetic properties rules (Lipinski et al., 1997 ; 2001) and results are summarized in the table 1. Fifteen compounds were synthesized and their physico-chemical properties are described in the table 2 .

Compounds were synthesized following the condensation reaction (figure 1).

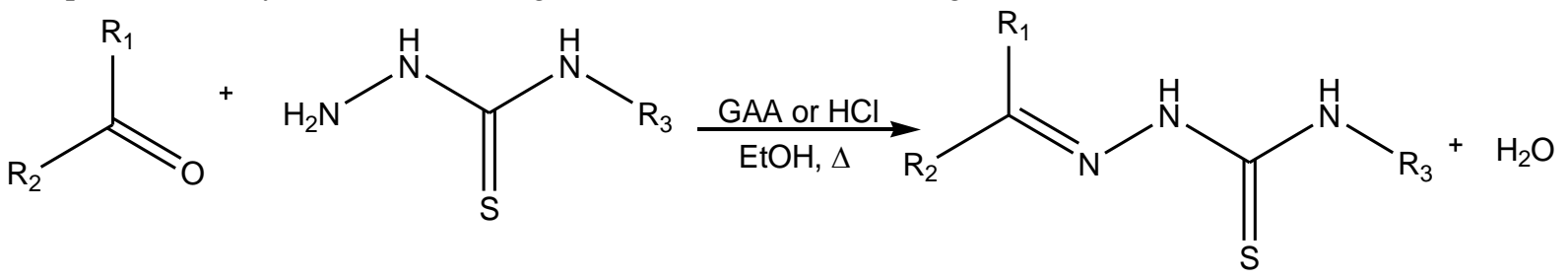

Figure 1:-Synthetic routes of thiosemicarbazones (scaffold).

Compounds : series $\mathrm{Bi}: \mathrm{R}_{1}=\mathrm{Et}, \mathrm{R}_{2}=\mathrm{Ph} ; \mathbf{B}_{\mathbf{1}} \mathrm{R}_{3}=\mathrm{H} ; \mathbf{B}_{\mathbf{2}} \mathrm{R}_{3}=\mathrm{Me}, \mathbf{B}_{\mathbf{3}} \mathrm{R}_{3}=\mathrm{Ph}$

Compounds : series $\mathrm{Ci}: \mathrm{R}_{1}=\mathrm{R}_{2}=\mathrm{Ph} ; \mathbf{C}_{\mathbf{1}} \mathrm{R}_{3}=\mathrm{H} ; \mathbf{C}_{\mathbf{2}} \mathrm{R}_{3}=\mathrm{Me}, \mathbf{C}_{\mathbf{3}} \mathrm{R}_{3}=\mathrm{Ph}$

Compounds : series Di : $\mathrm{R}_{1}=\mathrm{Me}, \mathrm{R}_{2}=\mathrm{p}-\mathrm{Me}-\mathrm{Ph} ; \mathbf{D}_{\mathbf{1}} \mathrm{R}_{3}=\mathrm{H} ; \mathbf{D}_{\mathbf{2}} \mathrm{R}_{3}=\mathrm{Me}, \mathbf{D}_{\mathbf{3}} \mathrm{R}_{3}=\mathrm{Ph}$

Compounds : series Ki : $\mathrm{R}_{1}=\mathrm{H}, \mathrm{R}_{2}=\mathrm{Ph} ; \mathbf{K}_{\mathbf{1}} \mathrm{R}_{3}=\mathrm{H} ; \mathbf{K}_{\mathbf{2}} \mathrm{R}_{3}=\mathrm{Ph}$ 
Compounds : series $\mathrm{Li}: \mathrm{R}_{3}=\mathrm{Ph} ; \mathbf{L}_{\mathbf{1}} \mathrm{R}_{1}=\mathrm{Ph} ; \mathrm{R}_{2}=\mathrm{o}-\mathrm{H}_{2} \mathrm{~N}-\mathrm{Ph} ; \mathbf{L}_{\mathbf{2}} \mathrm{R}_{1}=\mathrm{Ph} ; \mathrm{R}_{2}=\mathrm{o}-\mathrm{HO}-\mathrm{Ph} ; \mathbf{L}_{\mathbf{3}} \mathrm{R}_{1}=\mathrm{Ph}$;

$$
\mathrm{R}_{2}=\mathrm{p}-\mathrm{HO}-\mathrm{Ph} ; \mathbf{L}_{4} \mathrm{R}_{1}=\mathrm{Ph} ; \mathrm{R}_{2}=2-\mathrm{H}_{2} \mathrm{~N}-5-\mathrm{Cl}-\mathrm{Ph} \text {. }
$$

The structures of synthesized compounds were characterized with the TLC, spectrometrical analysis MS, IR, and especially with NMR ${ }^{13} \mathrm{C} \&{ }^{1} \mathrm{H}$.

Table 1:-Theoretical Pharmacokinetic and drug availability study

\begin{tabular}{|c|c|c|c|c|c|}
\hline Compounds & $\begin{array}{l}\text { Molecular weight } \\
\left(\mathrm{M} \text { in } \mathrm{g} \cdot \mathrm{mol}^{-1}\right)\end{array}$ & $\mathrm{C} \log \mathrm{P}$ & $\begin{array}{c}\text { Number of } \\
\text { H-bond donors }\end{array}$ & $\begin{array}{l}\text { Number of } \mathrm{H}- \\
\text { bond acceptors }\end{array}$ & $\begin{array}{c}\text { Number of criteria } \\
\text { met }\end{array}$ \\
\hline \multicolumn{2}{|c|}{\begin{tabular}{l|l} 
Rules & M $<500$
\end{tabular}} & $<5$ & $\leq 5$ & $<10$ & at least 3 \\
\hline $\mathbf{B}_{1}$ & 208.08 & 2.930 & 3 & 3 & all \\
\hline $\mathbf{B}_{2}$ & 221.09 & 2.816 & 2 & 3 & all \\
\hline $\mathbf{B}_{3}$ & 283.11 & 4.600 & 2 & 3 & all \\
\hline $\mathbf{C}_{1}$ & 255.09 & 4.102 & 3 & 3 & all \\
\hline $\mathrm{C}_{2}$ & 269.11 & 4.071 & 2 & 3 & all \\
\hline $\mathrm{C}_{3}$ & 331.11 & 5.400 & 2 & 3 & 3 \\
\hline $\mathbf{D}_{1}$ & 207.08 & 2.900 & 3 & 3 & all \\
\hline $\mathbf{D}_{2}$ & 221.09 & 2.786 & 2 & 3 & all \\
\hline $\mathbf{D}_{3}$ & 283.11 & 4.570 & 2 & 3 & all \\
\hline $\mathbf{K}_{1}$ & 179.24 & 1.865 & 3 & 3 & all \\
\hline $\mathbf{K}_{2}$ & 255.34 & 3.960 & 2 & 3 & all \\
\hline $\mathbf{L}_{1}$ & 346.43 & 4.673 & 4 & 4 & all \\
\hline $\mathbf{L}_{2}$ & 347.43 & 5.263 & 3 & 4 & 3 \\
\hline $\mathbf{L}_{3}$ & 347.43 & 5.263 & 3 & 4 & 3 \\
\hline $\mathbf{L}_{4}$ & 380.89 & 5.509 & 4 & 4 & 3 \\
\hline
\end{tabular}

Table 2:-Physico-chemical properties of synthesized compounds

\begin{tabular}{|c|c|c|c|c|}
\hline Compounds & Formula brute & $\begin{array}{c}{\left[\mathrm{MH}^{+}\right]^{\mathrm{a}} \text { in }} \\
\text { g.mol }^{-1}\end{array}$ & $\begin{array}{l}\text { Melting point } \\
\text { in }{ }^{\circ} \mathbf{C}\end{array}$ & $\begin{array}{l}\text { Yields } \\
\text { in \% }\end{array}$ \\
\hline $\mathbf{B}_{1}$ & $\mathrm{C}_{10} \mathrm{H}_{13} \mathrm{~N}_{3} \mathrm{~S}$ & 208.11 & 116-117 & 74 \\
\hline $\mathbf{B}_{2}$ & $\mathrm{C}_{11} \mathrm{H}_{15} \mathrm{~N}_{3} \mathrm{~S}$ & 222.06 & 98-99 & 67 \\
\hline $\mathbf{B}_{3}$ & $\mathrm{C}_{16} \mathrm{H}_{17} \mathrm{~N}_{3} \mathrm{~S}$ & 284.16 & $113-114$ & 80 \\
\hline $\mathrm{C}_{1}$ & $\mathrm{C}_{14} \mathrm{H}_{13} \mathrm{~N}_{3} \mathrm{~S}$ & 256.07 & $167-168$ & 84 \\
\hline $\mathbf{C}_{2}$ & $\mathrm{C}_{15} \mathrm{H}_{15} \mathrm{~N}_{3} \mathrm{~S}$ & 270.10 & $164-165$ & 52 \\
\hline $\mathrm{C}_{3}$ & $\mathrm{C}_{20} \mathrm{H}_{17} \mathrm{~N}_{3} \mathrm{~S}$ & 332.02 & $153-154$ & 82 \\
\hline $\mathbf{D}_{1}$ & $\mathrm{C}_{10} \mathrm{H}_{13} \mathrm{~N}_{3} \mathrm{~S}$ & 208.14 & $148-149$ & 81 \\
\hline $\mathbf{D}_{2}$ & $\mathrm{C}_{11} \mathrm{H}_{15} \mathrm{~N}_{3} \mathrm{~S}$ & 222.13 & $144-145$ & 71 \\
\hline $\mathbf{D}_{\mathbf{3}}$ & $\mathrm{C}_{16} \mathrm{H}_{17} \mathrm{~N}_{3} \mathrm{~S}$ & 284.07 & $175-176$ & 77 \\
\hline$K_{1}$ & $\mathrm{C}_{8} \mathrm{H}_{9} \mathrm{~N}_{3} \mathrm{~S}$ & 180.39 & $162-163$ & 65 \\
\hline$K_{2}$ & $\mathrm{C}_{14} \mathrm{H}_{13} \mathrm{~N}_{3} \mathrm{~S}$ & 256.07 & 199-200 & 75 \\
\hline $\mathbf{L}_{1}$ & $\mathrm{C}_{20} \mathrm{H}_{18} \mathrm{~N}_{4} \mathrm{~S}$ & 347.39 & 136-137 & 73 \\
\hline $\mathbf{L}_{2}$ & $\mathrm{C}_{20} \mathrm{H}_{17} \mathrm{~N}_{3} \mathrm{OS}$ & 348.37 & $186-187$ & 57 \\
\hline $\mathbf{L}_{3}$ & $\mathrm{C}_{20} \mathrm{H}_{17} \mathrm{~N}_{3} \mathrm{OS}$ & 348.41 & $172-173$ & 65 \\
\hline $\mathbf{L}_{4}$ & $\mathrm{C}_{20} \mathrm{H}_{17} \mathrm{ClN}_{4} \mathrm{~S}$ & 381.87 & $143-144$ & 61 \\
\hline
\end{tabular}

a : mass obtained of MS 
Compounds in series $\mathbf{B}_{\mathrm{i}}$

Propiophenone thiosemicarbazone $\left(B_{1}\right)$<smiles>CC/C(=N\NC(N)=S)c1ccccc1</smiles>

${ }^{13}$ C NMR $\delta$ (DMSO-d d $\left._{6}, \mathrm{ppm}\right): 179.13 \& 178.76(\mathrm{C}=\mathrm{S}) ; 155.81 \& 152.97(\mathrm{C}=\mathrm{N}) ; 136.14 ; 132.92 ; 130.03 ; 129.96$; $129.81 ; 128.69 ; 126.76 ; 126.46(\mathrm{C}-\mathrm{Ar}) ; 31.54 \& 20.30\left(-\mathrm{CH}_{2}-\right) ; 10.73 \& 10.65\left(\mathrm{CH}_{3}\right) .{ }^{1} \mathbf{H}$ NMR $\delta\left(\mathrm{CDCl}_{3}, \mathrm{ppm}\right)$ : $8.85 \& 8.65$ (sd, 1H, C=NNH-); 7.70-7.20 (m, 10H, H-Ar); $6.70 \& 6.55$ (sd, 2H, CS-NH $)$ ) $2.83 \& 2.61$ (qd, $2 \mathrm{H},-$ $\left.\mathrm{CH}_{2}-\right) ; 1.23 \& 1.11\left(\mathrm{td}, 3 \mathrm{H}, \mathrm{CH}_{3}\right) . \mathbf{I R} v\left(\mathrm{KBr}, \mathrm{cm}^{-1}\right): 3406,3301,3203(\mathrm{NH})$; shoulder at $3200\left(\mathrm{NH}_{2}\right) ; 1600(\mathrm{C}=\mathrm{N})$; 1074, 1053, 840 (N-CS-N). MS m/z [MH $\left.{ }^{+}\right]: 208.11$.

Propiophenone 4-methyl-3-thiosemicarbazone $\left(\mathbf{B}_{2}\right)$<smiles>CC/C(=N\NC(=S)NC)c1ccccc1</smiles>

${ }^{13} \mathrm{C}$ NMR $\delta\left(\mathrm{CDCl}_{3}, \mathrm{ppm}\right): 178.99 \& 178.62(\mathrm{C}=\mathrm{S}) ; 154.50 \& 151.50(\mathrm{C}=\mathrm{N}) ; 136.39 ; 133.17 ; 129.85 ; 129.72$;

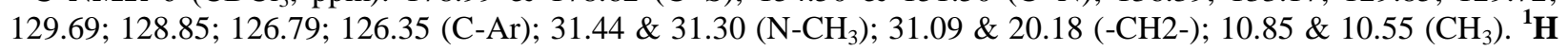
NMR $\delta\left(\mathrm{CDCl}_{3}, \mathrm{ppm}\right): 8.80 \& 8.50$ (sd, $\left.1 \mathrm{H}, \mathrm{C}=\mathrm{NNH}-\right) ; 7.17$ (q, 1H, $\left.\mathrm{CSNH}_{-} \mathrm{CH}_{3}\right) ; 7.80-7.30$ (m, 5H, H-Ar); $3.30 \&$ $3.20\left(\mathrm{sd}, 3 \mathrm{H}, \mathrm{N}-\mathrm{CH}_{3}\right) ; 2.70 \& 2.50\left(\mathrm{qd}, 2 \mathrm{H},-\mathrm{CH}_{2}-\right) ; 1.20 \& 1.10\left(\mathrm{td}, 3 \mathrm{H}, \mathrm{CH}_{3}\right)$. IR $v\left(\mathrm{KBr}, \mathrm{cm}^{-1}\right): 3414,3290(\mathrm{NH})$; $1544(\mathrm{C}=\mathrm{N}) ; 1062,1033,845(\mathrm{~N}-\mathrm{CS}-\mathrm{N})$. MS m/z [MH'] 222.06.

\section{Propiophenone 4-phenyl-3-thiosemicarbazone $\left(\mathrm{B}_{3}\right)$}<smiles>CC/C(=N\NC(=S)Nc1ccccc1)c1ccccc1</smiles>

${ }^{13}$ C NMR $\delta$ (DMSO-d 6 , ppm): $176.35 \& 176.01(\mathrm{C}=\mathrm{S}) ; 154.79 \& 152.02(\mathrm{C}=\mathrm{N}) ; 138.05 ; 137.96 ; 136.21 ; 133.06$; $130.08 ; 129.99 ; 129.85 ; 128.81 ; 126.83 ; 126.46 ; 126.13 ; 125.05 ; 124.21$ (C-Ar); $31.54 \& 20.45\left(-\mathrm{CH}_{2}-\right) ; 10.83$ \& $10.67\left(\mathrm{CH}_{3}\right) .{ }^{1} \mathbf{H}$ NMR $\delta\left(\mathrm{CDCl}_{3}, \mathrm{ppm}\right): 9.40(\mathrm{~s}, 1 \mathrm{H}, \mathrm{C}=\mathrm{NNH}-) ; 8.90 \& 8.60$ (nd, 1H, CSNH-Ph); 7.85-7.15 (m, $10 \mathrm{H}, \mathrm{H}-\mathrm{Ar}) ; 2.80 \& 2.65$ (qd, $\left.2 \mathrm{H},-\mathrm{CH}_{2}-\right) ; 1.30 \& 1.15$ (td, $\left.3 \mathrm{H}, \mathrm{CH}_{3}\right)$. IR $v\left(\mathrm{NaCl}, \mathrm{cm}^{-1}\right)$ : band 3450, $3294(\mathrm{NH})$; 1598, $1588(\mathrm{C}=\mathrm{N}) ; 1114,1055,920(\mathrm{~N}-\mathrm{CS}-\mathrm{N}) . \mathbf{M S} \mathrm{m} / \mathrm{z}\left[\mathrm{MH}^{+}\right]: 284.16$.

Compounds in series $\mathrm{C}_{\mathrm{i}}$

Benzophenone thiosemicarbazone $\left(\mathbf{C}_{1}\right)$<smiles>NC(=S)NN=C(c1ccccc1)c1ccccc1</smiles>

${ }^{13} \mathbf{C}$ NMR $\delta$ (DMSO-d $\left.{ }_{6}, \mathrm{ppm}\right): 179.05(\mathrm{C}=\mathrm{S}) ; 150.21(\mathrm{C}=\mathrm{N}) ; 136.50 ; 131.16 ; 128.54 ; 127.88$ (C-Ar). ${ }^{1} \mathbf{H}$ NMR $\delta$ $\left(\mathrm{CDCl}_{3}, \mathrm{ppm}\right): 8.60$ (s, 1H, C=NNH-); 7.60-7.20 (m, 10H, H-Ar); $6.42\left(\mathrm{~s}, 2 \mathrm{H}, \mathrm{CS}_{-} \mathrm{NH}_{2}\right) . \mathbf{I R} v\left(\mathrm{NaCl}, \mathrm{cm}^{-1}\right): 3410$, 3346, $3248\left(\mathrm{NH}_{2}\right) ; 3151(\mathrm{NH}) ; 1608(\mathrm{C}=\mathrm{N}) ; 1069,1026,846(\mathrm{~N}-\mathrm{CS}-\mathrm{N})$. MS m/z [MH']: 256.07. 
Benzophenone 4-methyl-3-thiosemicarbazone $\left(C_{2}\right)$<smiles>CNC(=S)NN=C(c1ccccc1)c1ccccc1</smiles>

${ }^{13} \mathbf{C}$ NMR $\delta\left(\mathrm{CDCl}_{3}, \mathrm{ppm}\right): 178.67(\mathrm{C}=\mathrm{S}) ; 149.57(\mathrm{C}=\mathrm{N}) ; 136.79 ; 131.46 ; 128.56 ; 127.72(\mathrm{C}-\mathrm{Ar}) ; 31.27\left(\mathrm{~N}-\mathrm{CH}_{3}\right)$. ${ }^{1}$ H NMR $\delta\left(\mathrm{CDCl}_{3}, \mathrm{ppm}\right): 8.65$ (s, $\left.1 \mathrm{H}, \mathrm{C}=\mathrm{NNH}-\right) ; 7.70$ (q, 1H, CSNH-CH $\left.\mathrm{CH}_{3}\right) ; 7.55-7.25$ (m, 10H, H-Ar); 3.25 (s, 3H, $\left.\mathrm{N}-\mathrm{CH}_{3}\right)$. IR $v\left(\mathrm{KBr}, \mathrm{cm}^{-1}\right): 3439,3315(\mathrm{NH}) ; 1531(\mathrm{C}=\mathrm{N}) ; 1073,1024,823(\mathrm{~N}-\mathrm{CS}-\mathrm{N}) . \mathbf{M S ~ m} / \mathrm{z}_{\left[\mathrm{MH}^{+}\right]: 270.10 .}$

Benzophenone 4-phenyl-3-thiosemicarbazone $\left(\mathrm{C}_{3}\right)$<smiles>S=C(NN=C(c1ccccc1)c1ccccc1)Nc1ccccc1</smiles>

${ }^{13}$ C NMR $\delta\left(\right.$ DMSO-d $\left.{ }_{6}, \mathrm{ppm}\right): 175.47(\mathrm{C}=\mathrm{S}) ; 149.22(\mathrm{C}=\mathrm{N}) ; 137,11 ; 135.73 ; 130.49 ; 129.65 ; 129.15 ; 128.02$, 127.75; 125.38 (C-Ar). ${ }^{1} \mathbf{H}$ NMR $\delta\left(\mathrm{CDCl}_{3}, \mathrm{ppm}\right): 9.45$ (s, 1H, C=NNH-); 8.75 (s, 1H, CSNH-Ph); 7.60-7.25 (m, 15H, H-Ar). IR v(NaCl, cm-1): 3338, $3304(\mathrm{NH}) ; 1594(\mathrm{C}=\mathrm{N})$; 1071, 1031, 855 (N-CS-N). MS m/z [MH']: 332.02.

Compounds in series $D_{i}$

4'-methylacetophenone thiosemicarbazone $\left(D_{1}\right)$<smiles>C/C(=N\NC(N)=S)c1ccc(C)cc1</smiles>

${ }^{13}$ C NMR $\delta$ (DMSO-d $\left.\mathrm{d}_{6}, \mathrm{ppm}\right): 179.12(\mathrm{C}=\mathrm{S}) ; 148.30(\mathrm{C}=\mathrm{N}) ; 140.29 ; 134.38 ; 130.45 ; 129.32 ; 126.44 ; 126.33(\mathrm{C}-$ $\mathrm{Ar}) ; 21.33\left(\mathrm{p}-\mathrm{CH}_{3}-\mathrm{Ar}\right) ; 13.62\left(\mathrm{CH}_{3}\right) .{ }^{1} \mathbf{H}$ NMR $\delta\left(\mathrm{CDCl}_{3}, \mathrm{ppm}\right): 8.85$ (s, $\left.1 \mathrm{H} \mathrm{C}=\mathrm{NNH}-\right) ; 7.60-7.15(\mathrm{~m}, 4 \mathrm{H}, \mathrm{H}-\mathrm{Ar})$; $6.62\left(\mathrm{~s}, 2 \mathrm{H}, \mathrm{CS}-\mathrm{NH}_{2}\right) ; 2.41\left(\mathrm{~s}, 3 \mathrm{H}, \mathrm{p}-\mathrm{CH}_{3}\right) ; 2.25\left(\mathrm{~s}, 3 \mathrm{H}, \mathrm{CH}_{3}\right)$. IR $v\left(\mathrm{NaCl}, \mathrm{cm}^{-1}\right): 3411,3379,3227\left(\mathrm{NH}_{2}\right) ; 3145$ $(\mathrm{NH}) ; 1598(\mathrm{C}=\mathrm{N}) ; 1093,1016,848(\mathrm{~N}-\mathrm{CS}-\mathrm{N}) ; 817,717\left(\mathrm{p}-\mathrm{CH}_{3}-\mathrm{Ar}\right) . \mathbf{M S} \mathrm{m} / \mathrm{z}\left[\mathrm{MH}^{+}\right]: 208.14$.

4'-methylacetophenone 4-methyl-3-thiosemicarbazone $\left(\mathrm{D}_{2}\right)$<smiles>CNC(=S)N/N=C(\C)c1ccc(C)cc1</smiles>

${ }^{13}$ C NMR $\delta\left(\mathrm{CDCl}_{3}, \mathrm{ppm}\right): 178.85(\mathrm{C}=\mathrm{S}) ; 146.89(\mathrm{C}=\mathrm{N}) ; 139.95 ; 134.64 ; 130.39 ; 129.29 ; 126.47 ; 126.26(\mathrm{C}-\mathrm{Ar})$; $31.27\left({\left.\mathrm{~N}-\mathrm{CH}_{3}\right)}_{3} ; 24.78 \& 21.32\left(\mathrm{p}-\mathrm{CH}_{3}\right) ; 13.50\left(\mathrm{CH}_{3}\right) .{ }^{1} \mathbf{H} \mathbf{N M R} \delta\left(\mathrm{CDCl}_{3}, \mathrm{ppm}\right): 8.70(\mathrm{~s}, 1 \mathrm{H}, \mathrm{C}=\mathrm{NNH}-) ; 7.20(\mathrm{q}, 1 \mathrm{H}\right.$,

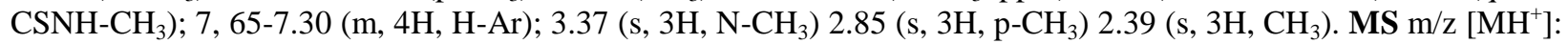
222.13 .

4'-methylacetophenone 4-phenyl-3-thiosemicarbazone $\left(\mathrm{D}_{\mathbf{3}}\right)$<smiles>C/C(=N\NC(=S)Nc1ccccc1)c1ccc(C)cc1</smiles> 
${ }^{13}$ C NMR $\delta$ (DMSO-d 6 , ppm): $176.25(\mathrm{C}=\mathrm{S}) ; 147.32(\mathrm{C}=\mathrm{N}) ; 140.33 ; 137.94 ; 134.44 ; 129.43 ; 128.89 ; 126.33$; 126.13; $124.22(\mathrm{C}-\mathrm{Ar}) ; 21.36\left(\mathrm{p}-\mathrm{CH}_{3}\right) ; 13.71\left(\mathrm{CH}_{3}\right) .{ }^{1} \mathbf{H}$ NMR $\delta\left(\mathrm{CDCl}_{3}, \mathrm{ppm}\right): 9.45(\mathrm{~s}, 1 \mathrm{H}, \mathrm{C}=\mathrm{NNH}-) ; 8.75(\mathrm{~s}, 1 \mathrm{H}$, CSNH-Ph); 7.70-7.25 (m, 9H, H-Ar); 2.42 (s, 3H, p-CH $)$; 2.35 (s, 3H, $\left.\mathrm{CH}_{3}\right)$. IR $v\left(\mathrm{NaCl}, \mathrm{cm}^{-1}\right)$ : band 3398-3299 $(\mathrm{NH}) ; 1633(\mathrm{C}=\mathrm{N}) ; 1100,1027,928(\mathrm{~N}-\mathrm{CSN}) ; 815,756\left(\mathrm{p}-\mathrm{CH}_{3}-\mathrm{Ar}\right) . \mathbf{M S} \mathrm{m} / \mathrm{z}[\mathrm{MH}+]: 284.07$.

\section{Compounds in series $\mathbf{K}_{\mathrm{i}}$}

Benzaldehyde-thiosemicarbazone $\left(K_{1}\right)$ :<smiles>NC(=S)N/N=C/c1ccccc1</smiles>

${ }^{13}$ C NMR $\delta$ (DMSO-d ${ }_{6}$ ppm): $177.97(\mathrm{C}=\mathrm{S}) ; 142.24(\mathrm{C}=\mathrm{N}) ; 127.26-134.15$ (aromatic C). ${ }^{1} \mathbf{H}$ NMR $\delta$ (DMSO-d $\mathrm{d}_{6}$ ppm): 7.39-7.79 (5H, several signals, Ar-H); $8.00\left(1 \mathrm{H}, \mathrm{s}, \mathrm{NH}_{2}\right) ; 8.06(1 \mathrm{H}, \mathrm{s}, \mathrm{CH}=\mathrm{N}) ; 8.21\left(1 \mathrm{H}, \mathrm{s}, \mathrm{NH}_{2}\right) ; 11.44(1 \mathrm{H}$, s, NH). IR $v\left(\mathrm{KBr} \mathrm{cm}^{-1}\right): 3401,3145(\mathrm{NH}) ; 1600,1584(\mathrm{C}=\mathrm{N}) ; 1217(\mathrm{C}=\mathrm{S}) . \mathbf{M S} \mathrm{m} / \mathrm{z}$ [MH $\mathrm{MH}^{+}$: 180.39.

\section{Benzaldehyde 4-phenyl-3-thiosemicarbazone $\left(\mathrm{K}_{2}\right)$}<smiles>S=C(N/N=C/c1ccccc1)Nc1ccccc1</smiles>

${ }^{13}$ C NMR $\delta\left(\right.$ DMSO-d $\left._{6}, \mathrm{ppm}\right): 176.00(\mathrm{C}=\mathrm{S}) ; 142.83(\mathrm{C}=\mathrm{N}) ; 139.04 ; 133.99 ; 130.01 ; 128.62 ; 127.61 ; 125.91 ;$ 125.31 (C-Ar). ${ }^{1} \mathbf{H}$ NMR $\delta\left(\mathrm{CDCl}_{3}, \mathrm{ppm}\right): 10.25(\mathrm{~s}, 1 \mathrm{H}, \mathrm{C}=\mathrm{NNH}-) ; 9.20(\mathrm{~s}, 1 \mathrm{H}, \mathrm{CSNH}-\mathrm{Ph}) ; 7.97(\mathrm{~s}, 1 \mathrm{H}, \mathrm{HC}=\mathrm{N})$; 7.65-7.25 (m, 10H, H-Ar). IR v(KBr, cm-1): band $2989(\mathrm{NH}) ; 1590(\mathrm{C}=\mathrm{N}) ; 1227(\mathrm{C}=\mathrm{S})$. MS m/z [MH $\left.{ }^{+}\right]: 256.32$.

\section{Compounds in series $\mathbf{L}_{\mathbf{i}}$}

2-aminobenzophénone 4-phenyl-3-thiosemicarbazone $\left(\mathrm{L}_{1}\right)$<smiles>Nc1ccccc1/C(=N/NC(=S)Nc1ccccc1)c1ccccc1</smiles>

${ }^{13} \mathrm{C}$ NMR $\delta\left(\mathrm{CDCl}_{3}, \mathrm{ppm}\right): 176.07(\mathrm{C}=\mathrm{S}) ; 143.74(\mathrm{C}=\mathrm{N}) ; 148.30 ; 137.91 ; 135.63 ; 131.76 ; 130.51 ; 129.96 ; 129.57$; 128.86; 127.58; 126.21; 124.33; 119.37; 116.80; $115.49(\mathrm{C}-\mathrm{Ar}) .{ }^{1} \mathbf{H}$ NMR $\delta\left(\mathrm{CDCl}_{3}\right.$. ppm): $10.30(\mathrm{~s}, 1 \mathrm{H}, \mathrm{C}=\mathrm{NNH}-)$; 8.87 (s, 1H, CSNH-Ph); 7.75-7.17 (m, 14H, H-Ar); $6.87 \& 6.70$ (s, 2H, Ar-NH $)_{2}$. MS m/z [MH'] : 347.39.

\section{2-hydroxybenzophenone 4-phenyl-3-thiosemicarbazone $\left(\mathbf{L}_{2}\right)$}<smiles>Oc1ccccc1/C(=N/NC(=S)Nc1ccccc1)c1ccccc1</smiles>

${ }^{13} \mathbf{C}$ NMR $\delta\left(\mathrm{CDCl}_{3} . \mathrm{ppm}\right): 176.03(\mathrm{C}=\mathrm{S}) ; 148.26(\mathrm{C}=\mathrm{N}) ; 154.56 ; 138.92 ; 133.12 ; 131.67 ; 129.77 ; 129.66 ; 128.53$; $128.23 ; 127.66 ; 126.10 ; 125.75 ; 124.05 ; 117.69 ; 116.53(\mathrm{C}-\mathrm{Ar}) .{ }^{1} \mathbf{H}$ NMR $\delta\left(\mathrm{CDCl}_{3} . \mathrm{ppm}\right): 10.50(\mathrm{~s}, 1 \mathrm{H}, \mathrm{OH}) ; 10.21$ (s, 1H, C=NNH-); 8.70 (s, 1H, CSNH-Ph); 7.80-7.00 (m. 14H. H-Ar). SM m/z [MH'] : 348.41. 


\section{4-hydroxybenzophenone 4-phenyl-3-thiosemicarbazone $\left(L_{3}\right)$}<smiles>Oc1ccc(/C(=N/NC(=S)Nc2ccccc2)c2ccccc2)cc1</smiles>

${ }^{13} \mathrm{C}$ NMR $\delta\left(\mathrm{CDCl}_{3} . \mathrm{ppm}\right): 175.75(\mathrm{C}=\mathrm{S}) ; 150.33(\mathrm{C}=\mathrm{N}) ; 159.04 ; 136.72 ; 131.70 ; 130.00 ; 129.89 ; 129.79 ; 128.30$; 128.25; 127.07; 125.95; 125;60; 121.34; 116.48 (C-Ar). ${ }^{1} \mathbf{H}$ NMR $\delta\left(\mathrm{CDCl}_{3} . \mathrm{ppm}\right): 10.30$ (s. $\left.1 \mathrm{H} . \mathrm{OH}\right) ; 9.90$ (s. $1 \mathrm{H}$. $\mathrm{C}=\mathrm{NNH}^{-}$); 8.80 (s. 1H. CSNH-Ph) ; 7.70-6.70 (m. 14H. H-Ar). MS m/z [MH'] $: 348.41$.

2-amino-5-chlorobenzophenone 4-phenyl-3-thiosemicarbazone $\left(\mathrm{L}_{4}\right)$<smiles>Nc1ccc(Cl)cc1/C(=N/NC(=S)Nc1ccccc1)c1ccccc1</smiles>

${ }^{13}$ C NMR $\delta$ (DMSO-d 6 . ppm): $176.79(\mathrm{C}=\mathrm{S}) ; 150.47(\mathrm{C}=\mathrm{N}) ; 144.20 ; 136.95 ; 132.08 ; 131.51 ; 131.29 ; 129.86$; 129.56; 128.75; 126.18; 124.38; 118.90; $116.98(\mathrm{C}-\mathrm{Ar}) .{ }^{1} \mathbf{H}$ NMR $\delta\left(\mathrm{CDCl}_{3} . \mathrm{ppm}\right): 10.30(\mathrm{~s}, 1 \mathrm{H}, \mathrm{C}=\mathrm{NNH}-) ; 9.00(\mathrm{~s}$, 1H, CSNH-Ph); 7.70-6.90 (m, 13H, H-Ar); 6.78 \& 6.35 (s, 2H, Ar-NH ${ }_{2}$. SM m/z [MH'] : 381.87.

\section{Pharmacology}

The antiparasitic activity of products was evaluated on the strain 427 of Trypanosoma brucei brucei using lapachol as witness. The toxicity activity of antitrypanosomal compounds was screened on Artemia salina Leach. The results of biological activities of products were obtained and expressed in $\mathrm{IC}_{50}$ and $\mathrm{LC}_{50}$ respectively. Selectivity of actives products are determined (table 3 ).

Table 3:-Trypanocidal and toxicity activity of synthesized compounds

\begin{tabular}{|c|c|c|c|c|c|c|}
\hline Compounds & $\mathrm{IC}_{50}(\mu \mathrm{M})$ & $\begin{array}{c}\text { Trypanocidal } \\
\text { activity }\end{array}$ & $\mathrm{LC}_{50}(\mu \mathrm{M})$ & Toxicity & $\begin{array}{c}\mathrm{SI}_{50} / \mathrm{IC}_{50} \\
\mathbf{L}_{\mathbf{1}}\end{array} \mathbf{L C}$ Selectivity \\
\hline $\mathbf{B}_{\mathbf{2}}$ & $87.15 \pm 1.13$ & low or no & - & - & - & - \\
\hline $\mathbf{B}_{\mathbf{3}}$ & $7.63 \pm 1.27$ & trypanocidal & $909.18 \pm 0.17$ & no toxic & 119.15 & selective \\
\hline & & & & & & \\
\hline $\mathbf{C}_{\mathbf{1}}$ & $67.17 \pm 3.15$ & moderate & $33.72 \pm 0.04$ & toxic & 0.50 & no selective \\
\hline $\mathbf{C}_{\mathbf{2}}$ & $23.27 \pm 1.24$ & moderate & $425.65 \pm 0.43$ & no toxic & 18.29 & selective \\
\hline $\mathbf{C}_{\mathbf{3}}$ & $8.48 \pm 0.89$ & trypanocidal & $366.76 \pm 0.02$ & no toxic & 43.25 & selective \\
\hline & & & & & & \\
\hline $\mathbf{D}_{\mathbf{1}}$ & $8.73 \pm 0.63$ & trypanocidal & $317.52 \pm 0.13$ & no toxic & 36.37 & selective \\
\hline $\mathbf{D}_{\mathbf{2}}$ & $5.42 \pm 1.03$ & trypanocidal & $185.31 \pm 1.09$ & toxic & 34.19 & selective \\
\hline $\mathbf{D}_{\mathbf{3}}$ & $>100$ & low or no & - & - & - & - \\
\hline & & & & & & \\
\hline $\mathbf{K}_{\mathbf{1}}$ & $>100$ & low or no & - & - & - & - \\
\hline $\mathbf{K}_{\mathbf{2}}$ & $>100$ & low or no & - & - & - & - \\
\hline & & & & & & \\
\hline $\mathbf{L}_{\mathbf{1}}$ & $2.83 \pm 0.17$ & trypanocidal & $42.50 \pm 1.19$ & toxic & 15.01 & selective \\
\hline $\mathbf{L}_{\mathbf{2}}$ & $3.86 \pm 0.86$ & trypanocidal & $14.55 \pm 1.32$ & toxic & 3.76 & selective \\
\hline $\mathbf{L}_{\mathbf{3}}$ & $2.76 \pm 1.00$ & trypanocidal & $5.56 \pm 0.63$ & toxic & 2.01 & selective \\
\hline $\mathbf{L}_{\mathbf{4}}$ & $12.16 \pm 0.44$ & moderate & $13.62 \pm 2.17$ & toxic & 1.12 & selective \\
\hline
\end{tabular}




\section{Discussion:-}

Synthetic molecules have physical properties compatible with reasonable pharmacokinetics and drug availability. The scaffold (Figure 1) has advantageous properties: low molecular weight, reasonable C.logP, good hydrogen bond donating and accepting capabilities (Table 1), easy and economical synthetic routes (Lipinski et al., 1997, 2001). The analysis of spectrometrical data gave especially in ${ }^{13} \mathrm{C}$ NMR spectra, peaks of $\mathrm{C}=\mathrm{S}$ from 179.13 to $175.47 \mathrm{ppm}$ and of $\mathbf{C}=\mathbf{N}$ between 150.47 and 143.74 ppm in all molecules. All aromatics carbons of the compounds ranged from 159.04 to $115.49 \mathrm{ppm} .{ }^{1} \mathrm{H}$ NMR spectra gave the characteristic protons in each structure: signals of protons $(\mathbf{H C}=\mathrm{N})$ were observed between 8.06 and $7.97 \mathrm{ppm}$ (products $\mathbf{K}_{\mathbf{1}} \& \mathbf{K}_{\mathbf{2}}$ ), we generally noted the disappearance of the peaks for the proton $\mathrm{NH} 2$ of thiosemicarbazide, except the protons in the amino group $\mathrm{H}_{2} \mathrm{~N}$ in molecules $\mathbf{L}_{\mathbf{1}}$ and $\mathbf{L}_{\mathbf{4}}$ respectively were identified at 6.87 and 6.70 and 6.78 and $6.35 \mathrm{ppm}$. In the products $\mathbf{L}_{2}$ and $\mathbf{L}_{3}$, the signal of the typical proton $\mathrm{HO}$ was observed respectively at 8.70 and $8.80 \mathrm{ppm}$. Aromatics protons in the compounds were obtained between 7.80 and $6.70 \mathrm{ppm}$. It is worth mentioning that substituents used here, $\mathrm{OH}$ and $\mathrm{NH}_{2}$, are both electron donating in the ortho and para positions. The analysis of these spectral data further confirms the structure of each molecule synthesized. In mass spectrometry, mass of each molecular ion peak (parent peak) obtained is very consistent and comparable to the theoretically estimated mass.

Pharmacological tests (table 3) showed that compounds presented interesting activity. On the parasites, some molecules $\mathbf{B}_{\mathbf{3}}, \mathbf{C}_{\mathbf{3}}, \mathbf{D}_{\mathbf{1}}, \mathbf{D}_{\mathbf{2}}$ and $\mathbf{L}_{\mathbf{1 - 3}}$ revealed a high trypanocidal effect with their $\mathrm{IC}_{50}$ between 2.76 and $8.73 \mu \mathrm{M}$ (IC $\mathrm{C}_{50}$ $<10 \mu \mathrm{M})$. Product $\mathbf{B}_{2}, \mathbf{C}_{\mathbf{1}}, \mathbf{C}_{\mathbf{2}}$ and $\mathbf{L}_{\mathbf{4}}\left(\mathrm{IC}_{50}=12<\mathrm{IC}_{50}<90 \mu \mathrm{M}\right)$ inhibited a moderate trypanocidal activity. The other compounds showed little or no activity because presented a high value of $\mathrm{IC}_{50}(>100 \mu \mathrm{M})$. These results are consistent with the scale of trypanocidal activity established in the previous works (Du et al., 2002 ; Greenbaum et al., 2004 ; Fujii et al., 2005). According their previous studies, thiosemicarbazones are trypanocidal when their IC $_{50}$ values are lower than $10 \mu \mathrm{M}$, and are regarded as moderate anti-trypanosomal agents if these values are between 10 and $100 \mu \mathrm{M}$, and have little or no activity when their $\mathrm{IC}_{50}$ are higher than $100 \mu \mathrm{M}$.

\section{Lipophilic and structure-activity relationship of compounds}

It has been reported in the literature that $\mathrm{N}(4)$-alkyl or $\mathrm{N}(4)$-arylthiosemicarbazones exhibit greater antitrypanosomal activity than their unsubstituted analogs, probably due to their increased lipophilicity (Pandeya et al., 2000 ; Beraldo and Gambino 2004 ; Perez-Robellodo et al., 2008). In our work, this is valid for the same series of compounds (table 4).

The data in the table show that for these three series of compounds, the most active are N(4)-methyl- and especially $\mathrm{N}(4)$-phenyl-3-thiosemicarbazones. Indeed, the N(4)-phenyl-3-thiosemicarbazones of propiophenone $\mathbf{B}_{\mathbf{3}}(7.63 \mu \mathrm{M})$ and benzophenone $\mathbf{C}_{\mathbf{3}}(8.48 \mu \mathrm{M})$, all trypanocides, are more active than their analogs $\mathrm{N}(4)$-methyl-3thiosemicarbazones $\mathbf{B}_{2}(87.15 \mu \mathrm{M})$ and $\mathbf{C}_{2}(23.27 \mu \mathrm{M})$ which presented moderate activity; they are also more active than unsubstituted thiosemicarbazones $\mathbf{B}_{\mathbf{1}}(>100 \mu \mathrm{M})$ and $\mathbf{C}_{\mathbf{1}}(67.17 \mu \mathrm{M})$. The same remark is made at the level of compounds $\mathbf{D}_{\mathbf{2}}$ and $\mathbf{D}_{\mathbf{1}}$, all trypanocides, where 4'-methylacetophenone N(4)-methyl-3-thiosemicarbazone $\mathbf{D}_{\mathbf{2}}$ (5.42 $\mu \mathrm{M})$ inhibited more trypanocidal activity than the unsubstituted thiosemicarbazone $\mathbf{D}_{\mathbf{1}}(8.73 \mu \mathrm{M})$.

Lipophilicity is a main physico-chemical determinant influencing the bioavailability, permeability and frequently the toxicity of drugs (Lipinski et al., 2001). A substance is all the more lipophilic as $\log \mathrm{P}$ is positive. The calculation of the $\log \mathrm{P}(\mathrm{C} \log \mathrm{P})$ involves the additivity rules of the hydrophobic constants of Rekker (Rekker, 1977). The higher the $\log \mathrm{P}(\mathrm{C} \log \mathrm{P})$ the lower $\mathrm{IC}_{50}$ and the more active substance (Du et al., 2002 ; Fujii et al., 2005).

For each of these series, it is noted that in general the introduction of the alkyl or aryl group on the nitrogen $\mathrm{N}(4)$ induces an increase in lipophilicity and also activity : $\mathbf{B}_{\mathbf{3}}$ and $\mathbf{C}_{\mathbf{3}}$ with $\mathbf{C l o g} \mathbf{P}=4.600$ and 5.400 respectively are all trypanocidal. In the Di series, the two trypanocidal compounds have almost the same degree of lipophilicity.

In the $\mathbf{L}_{1-4}$ series of 4-phenyl-3-thiosemicarbazones of the benzophenone derivatives, a significant increase in activity is also seen. Among these molecules, 4-hydroxybenzophenone 4-phenyl-3-thiosemicarbazone $\left(\mathbf{L}_{\mathbf{3}}\right)$ is the most active displaying an $\mathrm{IC}_{50}$ of $2.76 \mu \mathrm{M}$. A comparative study between 4-phenyl-3-thiosemicarbazones of benzophenone $\mathbf{C}_{\mathbf{3}}$ and its $\mathbf{L}_{\mathbf{1 - 3}}$ derivatives shows that the substitution of an ortho or para proton of a phenyl radical of benzophenone by an amino or a hydroxy group significantly enhanced trypanocidal activity $\left(\right.$ Table 3 ). The IC T0 $_{5}$ values found for the substituted $\mathbf{L}_{\mathbf{1 - 3}}$ derivatives are still low compared to that of the unsubstituted $\mathbf{C}_{\mathbf{3}}$ compound $\left(\mathrm{IC}_{50}=8.48 \mu \mathrm{M}\right)$. It appears that it is the substitution of the hydroxyl group in para position $\mathbf{L}_{2}$ which gives the best activity $\left(\mathrm{IC}_{50}=2.76 \mu \mathrm{M}\right)$. This group enriches its nucleus by its mesomeric donor effect. The ortho amino and 
hydroxy benzophenone derivatives are also more active than $\mathbf{C}_{\mathbf{3}}$. The difference in activity between these $\mathbf{L}_{\mathbf{1}}$ and $\mathbf{L}_{\mathbf{2}}$ molecules could be explained by the effect of the amino group or the substituted hydroxy group on the phenyl radical. Between ortho and para hydroxy-substituted products, it is para the most active $\mathbf{L}_{\mathbf{2}}$. This could be explained by the steric $\mathrm{OH}$ effect in the ortho position. There is a slight decrease in activity $\left(\mathrm{IC}_{50} \mathrm{p}-\mathrm{OH}=2.76\right.$ and $\mathrm{o}-\mathrm{OH}=$ $3.86 \mu \mathrm{M})$ (Du et al., 2002).

In our study, we have remarked that the substitution of the imine's hydrogen $\mathrm{CH}=\mathrm{N}$ of benzaldehyde 4-phenyl-3thiosemicarbazone $\left(\mathbf{K}_{\mathbf{2}}\right)$ by the radical ethyl (propiophenone $\mathbf{B}_{\mathbf{3}}$ ) and phenyl (benzophenone $\mathbf{C}_{\mathbf{3}}$ ) induced a trypanocidal activity. This observation confirms the work of $\mathrm{Du}$ et al. (2002). The $\mathrm{IC}_{50}$ value goes from more than $300 \mu \mathrm{M}$ (for $\mathbf{K}_{2}$ ) to $8.48 \mu \mathrm{M}$ (for $\mathbf{C}_{\mathbf{3}}$ ) and $7.63 \mu \mathrm{M}$ (for $\mathbf{B}_{\mathbf{3}}$ ). The last two compounds are also the most lipophilic (table 1).

Table 4:-Evolution of the trypanocidal activity with the thiosemicarbazone N(4)-alkyl or aryl substituted.

\begin{tabular}{|l|c|c|c|}
\hline \multicolumn{1}{|c|}{ Compounds } & $\mathbf{C} \log \mathbf{P}$ & $\mathbf{I C}_{\mathbf{5 0}}$ en $\boldsymbol{\mu} \mathbf{M}$ & Activity \\
\hline Propiophenone 4-phenyl-3-thiosemicarbazone $\mathbf{B}_{\mathbf{3}}$ & $\mathbf{4 . 6 0 0}$ & 7.63 & trypanocidal \\
\hline Propiophenone 4-methyl-3-thiosemicarbazone $\mathbf{B}_{\mathbf{2}}$ & 2.816 & 87.15 & moderate \\
\hline Propiophenone thiosemicarbazone $\mathbf{B}_{\mathbf{1}}$ & 2.930 & 210.00 & low \\
\hline Benzophenone 4-phenyl-3-thiosemicarbazone $\mathbf{C}_{\mathbf{3}}$ & $\mathbf{5 . 4 0 0}$ & 8.48 & trypanocidal \\
\hline Benzophenone 4-methyl-3-thiosemicarbazone $\mathbf{C}_{\mathbf{2}}$ & 4.071 & 23.27 & moderate \\
\hline Benzophenone thiosemicarbazone $\mathbf{C}_{\mathbf{1}}$ & 4.102 & 67.17 & moderate \\
\hline 4'-methylacetophenone 4-methyl-3-thiosemicarbazone $\mathbf{D}_{\mathbf{2}}$ & 2.786 & 5.42 & trypanocidal \\
\hline 4'-methylacetophenone thiosemicarbazone $\mathbf{D}_{\mathbf{1}}$ & 2.900 & 8.73 & trypanocidal \\
\hline
\end{tabular}

We then studied the larval toxicity of the active compounds on the trypanosome using the cytotoxicity of the lapachol $\left(\mathrm{LC}_{50}=281 \mu \mathrm{M}\right)$ as referred (Santos et al., 2003 ; Graminha et al., 2008). Shrimp larvae were selected in this study as biological model. Our different $\mathrm{LC}_{50}$ values obtained using the synthesized products presented toxic or no activity than lapachol. Among compounds, it is $\mathbf{B}_{\mathbf{2}}, \mathbf{C}_{\mathbf{1}}, \mathbf{D}_{\mathbf{2}}$ and the series $\mathbf{L}_{\mathbf{1 - 4}}$ which showed toxic activity, particularly the 4-phenyl-3-thiosemicarbazones of benzophenone derivatives $\mathbf{L}_{1-4}$ (Table 3 ). Indeed, there is a correlation between the toxicity of the compounds on shrimp larvae and their cytotoxicity on 9KB and 9PS cells (human carcinoma nasopharygien) (Pelka et al., 2000), and on A-549 cells of lung carcinoma and HT-29 cells of colon carcinoma (Carballo et al., 2002). In addition, we noticed that the most strongly trypanocidal compounds were also the most cytotoxic. With their $\mathrm{LC}_{50}$ and $\mathrm{IC}_{50}$ values, we have determined the selectivity indexe of each trypanocidal compound. Except $\mathbf{C}_{\mathbf{1}}$ all products displayed greater selectivity, especially $\mathbf{B}_{\mathbf{3}}$ with $\mathrm{SI}=119$ and compounds $\mathbf{C}_{2}, \mathbf{C}_{\mathbf{3}}, \mathbf{D}_{2}, \mathbf{D}_{\mathbf{1}}$ and $\mathbf{L}_{\mathbf{1}}(\mathrm{SI}>10)$. These results are in perfect agreement with the work of Tiuman et al., (2005) in which if the SI value obtained is greater than unity, the test compound is considered to be selective on the parasite and if SI value is less than unity, the test compound is more cytotoxic than anti-parasitic.

\section{Conclusion:-}

In this work, fifteen thiosemicarbazones and derivatives were synthesized and studied. Their biological activities were evaluated and products showed interesting trypanocidal activity on the parasite study and were selective. Some factors including lipophilicity, steric and electronic effects of the substituents have played a vital role in this activity. This study could open an interesting opportunity to the treatment of the trypanomiasis sickness.

\section{Acknowledgments:-}

This study was made possible thanks to the collaboration of the staff and material of the laboratories, IREMPTCBRSI-BENIN, LDRI-UCL-Belgium, that we thank very sincerely.

\section{References:-}

1. Soates, O.A.R., Echevarria, A., Bellieny, S.S.M., Pinho, T.R., de Leo, M.M.R., Seguins, A.W., Machado, M.G., Canto-Cavalheiro, M.M., Leon, L.L. (2011). Evaluation of thiosemicarbazones and semicarbazones as potential agents anti-Trypanosoma cruzi. Experimental Parasitology. 129(4): 381-387.

2. Rogolino, D., Bacchi, A., De Luca, L., Rispoli G, Sechi, M., Stevaert, A., Naesens, L., Carcelli, M. (2015). Investigation of the salicylaldehyde thiosemicarbazone scaffold for inhibition of influenza virus PA endonuclease. Journal of Biologiocal Inorganic Chemistry. 20: 1109-1121. doi: 10.1007/s00775-015-1292-0. 
3. Büscher, P., Cecchi, G., Jamonneau, V., Priotto, G. (2017). Human African trypanosomiasis. Lancet. 390: 2397409.

4. Zani, C., Bisceglie, F., Restivo, F.M., Feretti, D., Pioli, M., Degola, F., Montalbano, S., Galati, S., Pelosi, G., Viola, G.V.C., Carcelli, M., Rogolino. D., Ceretti, E., Buschini, A. (2017). A new approach using a battery of assays to evaluate the efficacy of thiosemicarbazone derivatives as antifungal and anti-mycotoxigenic agents and their cytotoxic and genotoxic activity. Food and Chemical Toxicology. 105: 498-505. doi: 10.1016/j.fct.2017.05.008.

5. Du, X., Guo, C., Hansell, E., Doyle, S.P., Caffrey, C.R., Holler, T.P., McKerrow, J.H., Cohen, F.E. (2002). Synthesis and Structure-Activity Relationship Study of Potent Trypanocidal thiosemicarbazone Inhibitors of the Trypanosomal Cysteine Protease Cruzain. Journal of Medicinal Chemistry. 45: 2695-2707.

6. Beraldo, H. and Gambino, D. (2004) Semicarbazones and thiosemicarbazones: their wide pharmacological profile and clinical applications. Química Nova. 27: 461-471.

7. Fujii, N., Mallari, J.P., Hansell, E.J., Mackey, Z., Doyle, P., Zhou, Y.M., Gut, J., Rosenthal, P.J., McKerrow, J.H., Guy, R.K. (2005). Discovery of potent thiosemicabazones inhibitors of rhodesain and cruzain. Bioorganic and Medicinal Chemistry. 15(1): 121-123.

8. Jeremy, P.M., Anang, S., Aaron, K., Conor, R.C., Michele, C., Fangyi, Z., James H.M., Guy, R.K. (2008). Discovery of trypanocidal thiosemicarbazone inhibitors of rhodesain and TbcatB. Bioorganic and Medicinal Chemistry Letters. 18(9): 2883-2885.

9. Simarro, P.P., Cecchi, G., Franco, J.R., Paone, M., Diarra, A., Ruiz-Postigo, J.A., Fèvre, M.E., Mattioli, C.R., Jannin, G.J. (2012). Estimating and mapping the population at risk of Sleeping Sickness. PLoS Neglected Tropical Diseases. 6(10) : e1859. doi: 10.1371/journal.pntd.0001859

10. Grant, C., Anderson, N. and Machila, N. (2015). Stakeholder Narratives on Trypanosomiasis, Their Effect on Policy and the Scope for One Health. PLoS Neglected Tropical Diseases. 9(12): e0004241. doi: 10.1371/journal.pntd.0004241

11. Amer, S., Ryu, O., Tada, C., Fukuda, Y., Inoue, N. et Nakai, Y. (2011). Molecular identification and phylogenetic analysis of Trypanosoma evansi from dromedary camels (Camelus dromedarius) in Egypt: a pilot study. Acta Tropica. 117(1): 39-46. doi: 10.1016/j.actatropica.2010.09.010.

12. Cox, A.P., Tosas, O., Tilley, A., Picozzi, K., Coleman, P., Hide, G. \& Welburn, S.C. ( 2010). Constraints to estimating the prevalence of trypanosome infections in East African zebu cattle. Parasite Vectors. 3: 82.

13. Baltz, T., Baltz, D., Giroud, C., Crockett, J. (1985). Cultivation in a semi defined medium of animal infective forms of Trypanosoma brucei, T. equiperdum, T. evansi, T. rhodhesiense et T. gambiense. EMBO Journal. 4(5): 1273-1277.

14. Räz, B., Iten, M., Grether-Bühler, Y., Kaminsky, R., Brun, R. (1997). The Alamar BlueTM assay to determine drugs sensitivity of African trypanosomes (Trypanosoma brucei rhodesiense and Trypanosoma brucei gambiense) in vitro. Acta Tropica. 68: 139-147.

15. Sleet, R.B., Brendel, K. (1983). Improved methods for harvesting and counting synchronous populations of Artemia nauplii for use in developmental toxicology. Ecotoxicology and Environmental Safety. 7: 435-446.

16. Abbott, W.A. (1925). A method of computing the effectiveness of an insecticide. Journal of Economic and Entomology 18: 265.

17. Hafner, E., Heiner, E., Noack, E. (1977). Mathematical analysis of concentration-response relationships. Arzneimittel-Forschung / Drug Research. 27: 1871-1873.

18. Lipinski, C.A., Lombardo, F., Dominy, B.W., Feeney, P.J. (1997) Experimental and computational approaches to estimate solubility and permeability in drug discovery and development settings. Advanced Drug Delivery Reviews. 23: 3-25.

19. Lipinski, C.A., Lombardo, F., Dominy, W.B., Feeney, J.P. (2001). Experimental and computational approaches to estimate solubility and permeability in drug discovery and development settings. Advanced Drug Delivery Reviews. 46: 3-26.

20. Greenbaum, D.C., Mache, Z., Hansell, E., Doyle, P., Gut, J., Caffrrey, C.R., Lehrman, J., Rosenthal, P.J., McKerrow, J.H., Chibale, K. (2004). Synthesis and structure activity relationships of parasiticidal thiosemicarbzone cysteine protease inhibitors against P. falciparum, T. brucei and T. cruzi. Journal of Medicinal Chemistry. 47(12):3212-3219.

21. Pandeya, S.N., Yogeeswari, P., Sausville, E.A., Mauger, A.B., Narayanan, V.L. (2000). Synthesis and antitumour activity of some aryl semicarbazones. Scientia Pharmaceutica. 68: 369-377.

22. Pérez-Rebolledo, A., Teixeira, L.R., Batista, A.A., Mangrich, A.S., Aguirre, G., Cerecetto, H., Gonzalez, M., Hernandez, P., Ferreira, A.M., Speziali, N.L., Beraldo, H. (2008). 4-nitroacetophenone derived 
thiosemicarbazones and their copper (II) complexes with significant in vitro antitrypanosomal activity. European Journal of Medicinal Chemistry. 43(5): 939-948.

23. Santos, P.L.P., Pinto, G.B., Takahashi, J.A., Silva, L.G.F., Boaventura, M.A.D. (2003). Biological screening of Annonaceous Brazilian medicinal plants using Artemia salina (Brine shrimp test). Phytomedicine. 10(2-3): 209212.

24. Graminha, A.E., Batista, A.A., Louro, S.R.W., Ziolli, R.L., Teixeira, L.R., Beraldo, H. (2008). 2Pyridinoformamide-derived thiosemicarbazones and their iron(III) complexes : potential antineoplastic activity. Polyhedron. 27: 547-551.

25. Pelka, M., Danzl, C., Distler, W., Petschelt, A. (2000). A new screening test for toxicity testing of dental materials. Journal of Dentistry. 28(5): 341-345.

26. Carballo, J.L., Hernàndez-Inda, Z.L., Perez, P., Garcia-Gravalos, D.C. (2002). A comparison between two brine shrimp assays to detect in vitro cytotoxicity in marine natural products. BMC Biotechnology. 2: 17. doi:10.1186/1472-6750-2-17

27. Tiuman, T.S., Ueda-Nakamura, T., Garcia Cortez, D.A., Dias Filho, B.P., Morgado-Diaz, J.A., de Souza, W., Nakamura, C.V. (2005). Antileishmanial activity of Parthenolide, a sesquiterpene lactone isolated from Tanacetum parthenium. Antimicrobial Agents chemother. 492: 176-182. 